\title{
Psicología de la Salud en el Proyecto San Carlos Borromeo de Barranquilla, Colombia
}

\section{Psychology of Health Project in San Carlos Borromeo of Barranquilla, Colombia}

\author{
Carmen Sierra Llamas ${ }^{1}$ \\ Universidad Simón Bolívar
}

\section{RESUMEN}

Introducción: El Proyecto San Carlos Borromeo es parte fundamental del programa académico de Psicología en Extensión y Proyección Social de la Universidad Simón Bolívar. Se aplica en el sector 5 del Suroccidente del Área Metropolitana de Barranquilla, específicamente en el Centro de Atención Integral a la Familia (CAIFAM). El énfasis del programa de Psicología de la Salud que allí funciona está en la importancia de mantener estilos de vida saludables, realizando talleres educativos de promoción, prevención e intervención en poblaciones atendidas. Objetivo: Sistematizar el programa de psicología de la salud en el proyecto San Carlos Borromeo desde el segundo semestre de 2005 hasta el 5 de diciembre de 2010. Materiales y métodos: Investigación de tipo cualitativa, con sistematización de experiencias, y con una evaluación a través de la lluvia de ideas y escalas de factores de riesgo. Resultados: Se atendieron 3047 personas, 1518 de género masculino y 1529 de género femenino. La población estuvo constituida por 929 niños, 1481 jóvenes, 456 adultos y 181 adultos mayores. Se realizaron cartillas sobre hipertensión y diabetes, y folletos sobre prevención al maltrato, prevención al abuso y al consumo de drogas, videos, entre otros. Conclusión: Aunque se lograron cambios importantes, es necesario el constante trabajo, debido a que la población está expuesta al manejo inadecuado de comportamientos saludables por la predominancia de un nivel socioeconómico bajo y la convivencia de familias numerosas, en su mayoría no nucleares.

Palabras Clave: psicología, salud, sistematización (Fuente: DeCS).

\begin{abstract}
Introduction: This paper focuses on Health Psychology programs. These programs are based on social and community work, which were carried out in San Carlos Borromeo Side 5 project from Barranquilla in its south-West Metropolitan Area, related to the Family Care Center (CAIFAM). The programs started in the second semester of 2005 and lasted until December 5, 2010. The emphasis of the health psychology program is to stress the importance of keeping healthy lifestyles, conducting educational workshops about promotion, prevention and intervention in populations during these six semesters. Objective: systematic program of health psychology at St. Charles Borromeo project. Materials and methods: A qualitative type, with systematization of experiences, with an evaluation through brainstorming and scales of risk factors. Results: Population and sample: 3,047 people served 1518 male and 1529 female. Distributed: 929 children, 1,481 youth, 456 adults and 181 elderly. Primers were achieved on hypertension and diabetes prevention brochures abuse, prevention and drug use, videos, and more. Conclusion: major changes could be achieved, but it constant work is necessary because people are exposed to inadequate management of healthy behaviors. Since the community is characterized by a low socioeconomic status, living with families, mostly non-nuclear.
\end{abstract}

Keywords: psychology, health, systematization (Source: MeSH, NLM).

Psicóloga Clínica. Magister en Psicología. Correo electrónico: csierra@unisimonbolivar.edu.co 


\section{Introducción}

El Estado y las instituciones privadas han diseñado políticas de atención cuyos beneficiados directos son los grupos a los que se dirigen. Es por esto que la Universidad Simón Bolívar, en el marco de las actividades de Extensión y Proyección Social, realiza diferentes programas encaminados a la construcción de un modelo de desarrollo social y humano para contribuir en el mejoramiento de la calidad de vida de las familias ubicadas en la Comuna 4, actualmente sector 5 de la Localidad Suroccidente.

\section{Reseña de la Localidad Suroccidente de Barranquilla}

La Oficina de Planeación dividió a Barranquilla en tres localidades: Norte, Suroriente y Suroccidente. La que corresponde al sector donde está ubicado CAIFAM y el Proyecto San Carlos Borromeo es la Localidad Suroccidente, la cual está conformada por 69 barrios, dos corregimientos (Juan Mina y La Playa) y un asentamiento de desplazados (Fundación Vida Digna).

La localidad está dividida en 6 sectores, ubicándonos en el sector 5, que comprende los barrios: Ciudad Modesto, El Pueblito, La Pradera, Olivos 1, 2, 3 (Los Ángeles), La Manga, Me Quejo, Por Fin, Las Terrazas, La Paz, Bernardo Hoyos, Pastoral Social, Las Estrellas, Villa del Rosario, Colinas Campestres, entre otros. Y cinco no legalizados: California, Loma Roja, Edén, Pinar del Río, Calamary (el cual pertenece a otro sector), los cuales son considerados en desventaja socioeconómica.

La administración local está conformada por el alcalde menor, la Junta de Acción Comunal, compuesta por 15 ediles, con su presidente, y también por los veedores de la comunidad, quienes recepcionan las quejas y se las hacen llegar a los alcaldes por medio de proyectos.

Por cada barrio, hay un promedio de tres grupos de tercera edad. En relación con el grupo de jóvenes, está conformado por adolescentes entre 12-18 años, ubicados en La Pradera, en la Iglesia San Carlos Borromeo y La Paz.

Geológicamente, todo el sector es inestable. La base es sobre todo tierra, suelo quebradizo y deslizante. Lo surcan tres arroyos (El Salao, La Paz y El Pueblito), los cuales dejan muchos damnificados en épocas de lluvia. En estos momentos, el arroyo El Salao está siendo canalizado por la administración distrital.

Otro problema ambiental que padece esta localidad es la laguna de oxidación (llegan los desechos de Barranquilla). Por falta de reforestación, se ha convertido en una fuente de olores putrefactos, situación que se agrava en el período invernal al viciar el aire del sector. Las estadísticas muestran las consecuencias negativas para la salud a causa de estas emanaciones, según lo reportado por médicos del Hospital de La Manga y del centro de salud ubicado en CAIFAM.

Por otra parte, las principales actividades económicas en la localidad son: la agricultura, con alrededor de un $15 \%$, siendo los principales productos yuca, papa, maíz, caña de azúcar, mango, mamón, ciruela y papaya. En este sentido, la producción no es tecnificada y es distribuida a pequeños compradores. Pero la principal actividad económica es el comercio, que abarca el $50 \%$ del total de la actividad económica del sector, concentrándose en bodegas pequeñas que venden al por mayor y al detal. Principalmente hay ventas de colchones, ferreterías, ventas de llamadas, misceláneas, estaciones de gasolina y moteles, entre otros negocios. También hay comerciantes que tienen microempresa y/o negocios pequeños, quienes realizan por sí mismos los productos que comercializan, pero la gran mayoría de ellos compran a terceros los insumos que distribuyen. Y, aunque sus productos son de buena calidad, los mercadean a bajo costo.

La economía informal abarca, por último, un 35\% y las personas que se emplean en esto trabajan en servicios generales y domésticos.

El total de habitantes era de 750.000, según el último censo del año 2005. Son personas que en general trabajan, pero la característica general de sus empleos es la inestabilidad laboral. Otras personas trabajan en empleos como vigilantes, camareras, domésticas, niñeras, madres comunitarias o en ventas ambulantes.

En general, los habitantes del sector distribuyen sus ingresos equitativamente, acorde con sus necesidades, pero la mayoría de las veces no alcanzan a cubrir la totalidad de sus gastos, porque ganan muy poco.

Las vías son de barro gallego. De éstas, solo se encuentran pavimentadas las que constituyen accesos principales, y funcionan como rutas de trasporte 
masivo. Los medios de trasporte utilizados son las motos y moto-taxis, además de buses y bicicletas. En los próximos meses entrará en circulación una ruta alimentadora y padrón de la empresa distrital Transmetro, que transitará por la Vía Circunvalar, aledaña al sector.

El sector cuenta con servicios de agua, alcantarillado, luz, gas, teléfono, predominando los teléfonos celulares. También cuenta con un hospital de Tercer Nivel, San Camilo, ubicado en el barrio La Manga, además de puestos de salud y consultorios particulares. En lo que se refiere a salud, la comunidad en general se encuentra amparada por el régimen subsidiado (SISBEN) y, en un porcentaje muy bajo, con el régimen contributivo. En cuanto a la vivienda, se construyen en mampostería, madera, bloques, con techos de eternit, teja, zinc, cartón, etc.

Respecto a la base cultural de la población, en su mayoría son personas con niveles aceptables de educación, dado que asistieron a un colegio, realizando o toda la primaria, el bachillerato o estudios técnicos. Un porcentaje bajo ha terminado estudios profesionales, predominando las áreas de la salud. No obstante, es importante anotar que existe un porcentaje, aunque mínimo, de analfabetismo en esta comunidad, especialmente en adultos mayores, quienes no han podido capacitarse porque hay entidades que llegan al barrio promocionando el programa de alfabetización, pero por muy corto tiempo y no culminan la meta con la población necesitada.

El 50\% de la población tiene un nivel de escolaridad básica primaria y el 35\%, de secundaria. Un 10\% son profesionales o técnicos, y el 5\% son analfabetas. En el sector hay 14 colegios oficiales, además de 72 hogares infantiles, distribuidos estos últimos en cuatro asociaciones.

\section{Características de la población}

Como se ha dicho, la población descrita hace parte del Suroccidente de Barranquilla. Y la población vinculada al programa de Psicología de la Salud está conformada por familias extensas, disfuncionales y multigeneracionales. En algunos casos, el liderazgo es patriarcal y, en otros, matriarcal, en su mayoría, madres cabeza de hogar. La interacción entre sus miembros es disfuncional, presentándose índices de maltrato físico y emocional. Así, muchas de estas familias presentan relaciones interpersonales deterioradas, en las que la agresividad es lo predominante. Del mismo modo, existen casos de sumisión y de dependencia económica, lo que hace que se incrementen los trastornos depresivos y ansiosos. Otro agravante de esta situación es la violación de los derechos de los menores, la baja autoestima y la presencia de esquemas negativos en alguno de sus miembros (1).

En este último sentido, los grupos muestran esquemas deteriorados en lo que concierne a la autoestima y el autoconcepto, deformados por situaciones que conllevan desde el maltrato emocional ocurrido en la infancia hasta la edad adulta, y por relaciones de parejas igualmente disfuncionales. Lo anterior genera estilos de afecto alterados, como el estilo dependiente débil, que trata de mantener la relación a toda costa y bajo cualquier circunstancia para evitar sentirse invalidado. Igualmente, se observa el estilo pasivo-agresivo, en el que la persona, por diversas razones, ha creado un conflicto profundo con la autoridad y no lo ha logrado solucionar (1).

El compromiso de los grupos de esta población en cuanto a crecimiento personal está basado en los valores, principalmente el valor a la vida, el cual se refiere al vitalismo o aspecto biológico. También están presentes los valores de utilidad, relacionados con el nivel económico en cuanto a bienes de consumo y satisfacción de necesidades básicas, como la vivienda.

Muestran creencias irracionales, tales como la necesidad de ser aprobado por los demás, auto exigencias y el culpar a otros de sus problemas. Asimismo, presentan signos de resentimiento, evidenciados en el hecho de culpar a los demás en lugar de asumir la responsabilidad de sus experiencias. Otro aspecto relevante son los pensamientos automáticos, unidos a los esquemas pasados de minusvalía, lo que trae como consecuencia estados melancólicos en algunas de estas personas.

En el estado de salud general, se detecta a grupos de jóvenes propensos a la diabetes. Al respecto, existen factores de riesgo que pueden incidir en que esta enfermedad crónica aparezca en estos jóvenes en cualquier momento, tales como antecedentes familiares, dietas incontroladas, estilos de vida inadecuados y falta de motivación para empezar a ser conscientes de esta problemática. 
En el caso de las personas hipertensas, que también hay en gran número, el objetivo del programa consiste en controlar la presión con el manejo adecuado de las emociones y de sus comportamientos, determinando para ello los factores de riesgo (2). En este sentido, se es necesario concientizar a la población sobre la importancia de adherirse al régimen terapéutico que se les prescriba. Pero, además, es fundamental que se incorporen a un régimen alimenticio para mantenerse en el peso adecuado, que, por ejemplo, realicen ejercicios diariamente; es decir, mejorar la calidad de vida (lo que significa cambiar de conductas, actitudes, creencias acerca de las prácticas saludables, de riesgo y enfermedades) con el manejo de comportamiento saludable. Este grupo de personas estudiadas sufre otras enfermedades como colesterol y triglicéridos altos.

Volviendo al tema de la psicología de la salud, la población de estudio utiliza estrategias de afrontamiento, especialmente la focalizada en la solución del problema, búsqueda de apoyo y la religión. A través de las capacitaciones dadas en psicología, en estos últimos semestres, ya los habitantes del sector 5 del Suroccidente de Barranquilla conocen la importancia de relajarse y lo fundamental que es el manejo de la respiración de una manera adecuada, además de detectar los pensamientos automáticos e irracionales que pueden incidir en las emociones, como la ira, actitud que genera en ellos pérdida de la estabilidad emocional.

Uno de los aspectos positivos de esta población es la motivación que tiene para recibir las capacitaciones, el entusiasmo hacia la vida y el marcado interés en detectar a tiempo, por ejemplo, si son hipertensos para aprender los correctivos que prevengan o mejoren esta enfermedad. En este sentido, la mayoría de los integrantes del grupo de hipertensos asiste a la Asociación Mis Años Bellos.

Enfocando otro aspecto de nuestro estudio, en general, se puede decir que la salud del grupo de mujeres lactantes y madres comunitarias es satisfactoria, aunque se observa sobrepeso en algunas de ellas. Es importante señalar que no asisten a un programa de ejercicios, pero sí acuden a los controles médicos en forma oportuna. Las enfermedades frecuentes entre ellas se relacionan con los procesos gripales.
La mayoría de la población es de bajos estratos (1, 2 ), contando con ingresos menores o iguales a un salario mínimo. En el caso del grupo de hipertensos se observa que muchos no están pensionados.

De otro lado, la población de la Comuna Cinco está organizada en colectivos infantiles, organizaciones juveniles y de mujeres, comedores populares, escuelas comunitarias, ciudadelas educativas, colegios, organizaciones de la sociedad civil, entre otros, que trabajan en el ámbito local, la cuadra, el barrio y la comuna, promoviendo espacios de encuentro y actividades que reivindican la necesidad del diálogo, el respeto mutuo, la solidaridad y la confianza entre los vecinos de la localidad. Con estos espacios, donde se construye ciudadanía, se encuentra comprometido el Hospital San Camilo y la Casa de Justicia "La Paz". Esta última es una institución garante de la presencia del Estado, que facilita el acceso a la justicia para los sectores populares, y cumple también las veces de entidad promotora de convivencia, de los derechos humanos y de los métodos alternativos de solución de conflictos, que, en una palabra, facilita la participación de la comunidad en el diagnóstico y solución de su problemática social.

Teniendo en cuenta las bases exploratorias de la localidad, la Universidad Simón Bolívar estableció un convenio con la Fundación San Carlos Borromeo con el objetivo de ayudar a las familias a lograr una mejor calidad de vida. Dicho estudio encontró problemas tales como violencia, pobreza, desempleo, problemas económicos, drogadicción, entre otras muchas dificultades derivadas de las anteriores.

Este proyecto, planificado conjuntamente con líderes de la comunidad y con agremiaciones sociales legitimadas, como son la ya citada Fundación San Carlos Borromeo y la parroquia del mismo nombre, se desarrolla gracias a los esfuerzos en pro del desarrollo de estos sectores. El proyecto nació en 1997, como iniciativa del programa de Psicología de la Universidad Simón Bolívar y la Fundación San Carlos Borromeo, lo que lo convierte en un programa de Extensión y Proyección Social de la Universidad.

El Centro de Atención Integral a la Familia (CAIFAM) es un ente surgido en desarrollo del Proyecto San Carlos Borromeo, ubicado en la localidad Suroccidente, sector 5, específicamente en el barrio Los Olivos-Primera Etapa. Es un sitio de prácticas donde concurren los estudiantes de las 
distintas facultades de la Universidad Simón Bolívar, con el objetivo de fortalecer el aprendizaje obtenido en las aulas de clases, y sirviendo a la comunidad con la aplicación de sus conocimientos. A ello se agrega el objetivo de profundizar desde la transdisciplinariedad, el estudio y comprensión del comportamiento humano, articulado con la naturaleza y el desarrollo humano; todo lo cual se logra con la formulación, la ejecución y la evaluación de programas de servicio social y de desarrollo social que articulen objetivos de investigación e intervención profesional. Programas como Psicología, Trabajo Social, Enfermería, Derecho, Fisioterapia, Educación, Administración e Ingeniería envían a sus estudiantes para que éstos orienten en sus prácticas, desde el área clínica, social y comunitaria (3), los conocimientos adquiridos en el transcurrir de sus estudios.

Acorde con todo lo propuesto hasta aquí, el objetivo del presente artículo consiste en sistematizar el programa de Psicología de la Salud en el proyecto Social San Carlos Borromeo, adscrito a las funciones de extensión y proyección social liderado por el Programa de Psicología de la Universidad Simón Bolívar, entre el segundo semestre del año 2005 y el segundo semestre de 2010.

\section{Materiales y Métodos}

La presente es una investigación cualitativa, con sistematización de experiencias. La sistematización como práctica investigativa se inscribe en una perspectiva "que busca comprender y tratar con lo cualitativo de la realidad" (4), es decir, su enfoque es más dialectico e interpretativo, para lo cual se recurre a lo descriptivo.

\section{Desarrollo de la Experiencia: La Sistematización}

Así como hay momentos para la planeación, la ejecución y la evaluación de las actividades, igualmente es necesario adecuar y dedicar un espacio para la sistematización de las mismas. De este modo, la sistematización se presenta como una alternativa a la evaluación tradicionalmente aplicada a los proyectos sociales y educativos, y como una reacción al positivismo predominante. Es también una respuesta a las insuficiencias de la investigación social, que invita a analizar las problemáticas que obstaculizan los proyectos de cambio y de intervención social (5).

Cienc. innov. salud. Junio 2013; 1 (1): 57 - 68. Universidad Simón Bolívar (Col). ISSN: 2344-8636 http://portal.unisimonbolivar.edu.co:82/rdigital/innovacionsalud
La revisión de artículos y ponencias sugiere que la sistematización, como concepto y práctica metodológica, no tiene un significado único. Por el contrario, gran parte de su riqueza radica en la diversidad de enfoques que se utilizan y que dan cuenta de la contextualización y sentido práctico otorgada a la reflexión de la experiencia.

En los proyectos educativos que son objeto de la sistematización y en las mismas experiencias de sistematización, no se realiza un análisis crítico de la teoría de la acción que subyace en las prácticas observadas. Por el contrario, en la mayor parte de las experiencias educativas, la acción social de los sujetos se entiende como un comportamiento derivado de estructuras o de variables, tenidas en cuenta en los talleres de sensibilización o capacitación encontradas por los encargados de la actividad (5), de acuerdo con los conocimientos y tipos de información que están al alcance de éstos. Este énfasis cognitivista entiende el cambio, principalmente, como una transformación en el terreno de los conocimientos, en las interpretaciones y en la información que el sujeto posee. En consecuencia, la educación y la intervención consistirán en transmitir adecuadamente nuevas interpretaciones para producir los cambios deseados en las prácticas de los sujetos (6).

Se piensa entonces que un cambio en los modos de pensar y en las informaciones que el sujeto posee, producirá los cambios deseados. Desde esta perspectiva, la transmisión y la enseñanza ocupan un lugar central. Pero, la evidencia empírica de numerosos proyectos educativos en diversos campos de acción social demuestra que el proceso no es tan simple. En efecto, las acciones de las personas y de las organizaciones no cambian como consecuencia automática del aumento de información, ni tampoco el proceso educativo funciona como una actividad instructiva y de transmisión vertical de saberes o de representaciones que van de un lado hacia otro.

Los profesionales y beneficiarios, al realizar sus interpretaciones, no aplican mecánicamente un saber pre-construido. Por el contrario, adecúan sus criterios al contexto y a la situación; anticipan e imaginan las posibilidades de interpretación del otro y cooperan con su interlocutor en la construcción de una racionalidad común (6), que les permite negociar y colaborar de acuerdo con causalidades y formas de pensar plausibles para el otro (7). 
No existe así el traspaso de un criterio o contenido de un lado a otro, sino, más bien, un proceso inferencial de interpretaciones recíprocas que adecúan y transforman los contenidos en el mismo acto de comunicación. De este modo, las categorías de interpretación no existen en forma ajena a las situaciones y a las subjetividades involucradas en la interacción. Éstas emergen y se realizan en el mismo curso de la interacción (6).

Desde esta perspectiva, las representaciones sociales deben entenderse como un medio de producción de la realidad en que se realiza el intercambio comunicativo. No se trata de ideas estáticas que entran en la cabeza desde fuera, sino de significaciones colectivas construidas a través de la práctica comunicativa. La representación se construye y sostiene en la interacción discursiva que el sujeto tiene en los distintos contextos sociales y comunicativos en los cuales participa (8).

De este modo, el estudio de estas interacciones, de los procesos de negociación de sentidos e interpretaciones y de los compromisos que los actores establecen, resultan claves para describir la intervención social y comprender sus resultados. En efecto, lo que importa no es la información que aparentemente pasó de un lado a otro, sino lo que ocurrió y se realizó en dicho intercambio, gracias a la conversación y al diálogo entre los interactuantes. En este intercambio, intervienen procedimientos de transacción y de negociación, a través de los cuales los interlocutores construyen acuerdos y compromisos en torno al objeto o problema que se aborda en la intervención o en la experiencia educativa.

Por todas estas circunstancias, hoy día hay consenso sobre la necesaria conexión de la sistematización y la investigación social. En efecto, la sistematización, más que entenderse como una alternativa que rechaza o niega la investigación, define una modalidad particular de investigación cuyo objeto es la acción social. Su preocupación es dar cuenta y describir esta acción.

\section{Psicología de la Salud}

Los aportes realizados por la Psicología de la Salud comprenden el análisis de posibles interacciones implicadas en el proceso del mantenimiento o promoción de la salud y a la prevención de la enfermedad.
La prevención se refiere a la adquisición de aprendizajes encaminados a la disminución de factores de riesgo propios de un estilo de vida insano. En la promoción, las acciones están dirigidas a la adquisición de hábitos saludables o de factores protectores de salud. Ambas metas forman parte de una estrategia de educación para la salud, entendida como una compleja combinación de métodos de aprendizaje dirigidos a la adopción de comportamientos útiles para mejorar o sostener la salud.

La prevención pertenece a ámbitos clínicos y, como ya se dijo, su objetivo es evitar una enfermedad. Por ejemplo, una vacuna previene una enfermedad particular. Por el contrario, la promoción de la salud está relacionada con los ámbitos sociales y apunta hacia el bienestar comunitario (9).

La adquisición de un comportamiento saludable o la modificación de un comportamiento es un proceso complejo que depende de muchos factores, los cuales incluyen desde la información sobre los comportamientos poco saludables hasta el diseño de estrategias de autocontrol para su adquisición, mantenimiento o recuperación en casos de recaída.

Según Flórez (10), la dimensión psicológica de la promoción / prevención, concebida como proceso, comprende las siguientes fases:

1) Conocimiento de los riesgos protectores.

2) Claridad sobre la propia exposición al riesgo de protección.

3) Intenciones de involucrarse en la modificación del riesgo/protección.

4) Toma de decisiones para modificar efectivamente el riesgo de protección.

5) Elaboración de planes de autocontrol o de manejo de contingencias.

6) Mantenimiento de las acciones.

7) Planes de recuperación en caso de recaídas.

El programa de Psicología de la Salud, en su fase exploratoria, se inició en el segundo semestre de 2005, en la Zona 5 del Suroccidente de la ciudad de Barranquilla, y se extendió hasta el 5 Diciembre de 2010 (2). 


\section{Descripción del programa}

El programa de Psicología de la Salud apunta hacia el fomento de estilos de vida saludable, y su objetivo consiste en diseñar e implementar programas que contribuyan a la promoción y prevención de la salud en relación a enfermedades crónicas o de otras disfunciones, que revisten gran importancia por sus niveles de incidencia en la población general. Lo anterior se logra motivando a las personas y los grupos a la práctica de estilos de vida saludables para contribuir a una mejor calidad de vida.

\section{Sus objetivos específicos son:}

- Brindar atención psicológica a la población que participa del programa, a partir de la evaluación del perfil cognitivo (esquemas, creencias, emociones, estrategias de afrontamiento) y su relación con la aceptación de la enfermedad y adherencia al tratamiento, para el manejo de los niveles de estrés.

- Fomentar el manejo de hábitos saludables en la población atendida, mediante talleres de sensibilización que permitan un mejor estilo de vida en la comunidad atendida.

- Promover la investigación desde la Psicología de la Salud, mediante la aplicación de instrumentos estandarizados en salud que permiten identificar los comportamientos saludables, para diseñar programas ajustados a la necesidad del entorno.

Este programa es liderado por el Programa de Psicología de la Universidad Simón Bolívar de Barranquilla, en el Centro de Atención Integral a la Familia (CAIFAM), y está dirigido a la comunidad de hipertensos, jóvenes propensos a desarrollar diabetes, madres comunitarias y mujeres lactantes y, en general, a toda la comunidad necesitada.

El programa está diseñado de manera que el aprendizaje sea vivencial, aspirando a que el arraigamiento de las competencias, aptitudes y actitudes referentes a la salud, sea más efectivo en la población beneficiada. En tal sentido, la metodología lúdica constituye una estrategia que garantiza el arraigamiento de los conceptos ya mencionados anteriormente. También, a través de la metodología participativa, se consulta, debate, reflexiona y se pone en común: son los ejes fundamentales para el trabajo a través de talleres con los grupos.

En conclusión, grosso modo, el programa busca que la acción de intervención psicosocial sea participativa en todo el sentido de la palabra. Para esto, en su realización se deben tener en cuenta las potencialidades de los grupos con los cuales se trabaja, de modo que se logren establecer nuevas herramientas para la mejora en los estilos de vida $\mathrm{Y}$ esas herramientas deben surgir de la comunidad, de acuerdo con las capacidades de ella.

\section{Resultados}

\section{Beneficiarios y beneficios de la comunidad}

Número total de beneficiarios. El total de las personas atendidas fue de 3047, de los cuales 1518 fueron hombres y 1529, mujeres. Clasificados así: 929 niños, 1481 jóvenes, 456 adultos y 181 adultos mayores.

Por otra parte, a lo largo de estos semestres, se han logrado realizar 80 talleres a la población beneficiada, entre cuyos temas se encuentran: Estrategias de afrontamiento, asertividad, relaciones interpersonales y manejo de conflictos, prevención al maltrato y al abuso sexual, manejo de una sexualidad sana, salud renal, prevención a enfermedades como la diabetes e hipertensión.

Con la metodología utilizada, se logró evaluar a la población utilizando medios que van desde la lluvia de ideas hasta escalas de factores de riesgo para diagnosticar riesgos prevalentes en la población. Entre los productos obtenidos, aparecen: historias clínicas, cartillas sobre hipertensión y diabetes, folletos sobre prevención al maltrato, prevención al abuso y al consumo de drogas, tres videos sobre las actividades del programa, 11 informes ejecutivos, con sus respectivas socializaciones a la facultad y a la comunidad, y fotos. 
Tabla 1. Población beneficiada del programa de Psicología de la Salud entre 2005-2 y 2010-2

\begin{tabular}{|c|c|c|c|c|c|c|c|c|c|c|c|}
\hline \multirow{3}{*}{$\begin{array}{c}\text { Población } \\
\text { beneficiada } \\
\text { por año }\end{array}$} & \multicolumn{8}{|c|}{ POBLACIÓN DIRECTA } & \multirow{3}{*}{ TOTAL } & \multirow{3}{*}{$\begin{array}{c}\text { POBLACIÓN } \\
\text { INDIRECTA } \\
\text { No. de personas }\end{array}$} & \multirow{3}{*}{$\begin{array}{c}\text { GRUPOS } \\
\text { ETARIOS }\end{array}$} \\
\hline & \multicolumn{2}{|c|}{ Niños } & \multicolumn{2}{|c|}{ Adolescentes } & \multicolumn{2}{|c|}{ Adultos } & \multicolumn{2}{|c|}{ Ancianos } & & & \\
\hline & $\mathbf{M}$ & F & $\mathbf{M}$ & $\mathbf{F}$ & M & $\mathbf{F}$ & M & F & & & \\
\hline 2005-II & & & & & & 15 & & & 15 & 60 & $20-28$ años \\
\hline 2006-I & & & & & & 22 & & & 22 & 88 & 20-25 años \\
\hline 2006-II & & & & & & & 2 & 13 & 15 & 60 & 56-70 años \\
\hline 2007-I & & & 13 & 22 & & & 2 & 11 & 48 & 192 & 13-70 años \\
\hline 2007-II & & & 5 & 13 & & & 2 & 7 & 27 & 108 & 12-75 años \\
\hline 2008-I & 30 & 6 & 13 & 24 & 1 & 31 & 11 & 23 & 139 & 5.166 & 5-73 años \\
\hline 2008-II & 48 & 43 & 68 & 71 & 5 & 49 & 4 & 20 & 308 & 1.232 & 2-72 años \\
\hline 2009-I & 60 & 74 & 208 & 204 & 15 & 69 & 6 & 15 & 651 & 2.596 & 5-72 años \\
\hline 2009-II & 59 & 22 & 90 & 90 & 12 & 51 & 10 & 16 & 350 & 1.400 & 5-73 años \\
\hline 2010-I & 194 & 122 & 232 & 161 & 44 & 37 & & & 790 & 3.160 & 2 a 55 años \\
\hline 2010-II & 187 & 84 & 155 & 112 & 23 & 82 & 19 & 20 & 682 & 2.708 & 3-82 años \\
\hline TOTAL & 578 & 351 & 784 & 697 & 100 & 356 & 56 & 125 & 3.047 & 16.770 & \\
\hline
\end{tabular}

Las habilidades adquiridas por parte de la comunidad fueron:

- -Respeto, no agresión y autoestima.

- -Reflexiones después de terminado el taller correspondiente. Todo esto para la formación de una cultura hacia la importancia de la salud.

- -El concepto de estilo de vida saludable y calidad de vida.

- -La importancia de ejercitar la memoria. Aprendizaje sobre los diferentes tipos de memoria.

- -La importancia del autoconcepto, la autoeficacia y la autoimagen en el crecimiento personal.

- -Detección de pensamientos deformados en el caso de los jóvenes propensos a desarrollar diabetes.

- -Destrezas en el reconocimiento de conductas inadecuadas en el manejo de la salud

- -Capacidad para expresar las inquietudes y comunicar los puntos de vista de manera constructiva.

- -Fomento y prevención a través de los estilos de vida saludables.

\section{Habilidades adquiridas por los estudiantes en sus prácticas}

- Aplicación de entrevistas e instrumentos de investigación y diagnóstico psicológico.

- -Habilidad para manejar grupos y realizar talleres a las diferentes poblaciones intervenidas.
- -Manejo de grupos de niños y diseño de actividades lúdicas.

- -Investigación y adaptación de los temas relacionados con la problemática del grupo.

- -Diseño de actividades que permitieron la sensibilización y la participación activa de la comunidad.

- -Sensibilización de la problemática a los grupos asignados.

- -Habilidades para el trabajo en equipo.

- -Aplicación de algunas técnicas de intervención comportamental como relajación, detección de pensamientos automáticos e ideas irracionales.

- -Investigación de una temática nueva, como en el caso de los estudiantes de VI semestre de Psicología en lo pertinente a memoria en adultos mayores y Psicología de la Salud.

- -Aplicación de un instrumento a una población numerosa.

- -Realización de una investigación en sentido estricto.

- -Programación de actividades de talleres bajo parámetros establecidos.

- -La gestión de formatos como la sistematización de las actividades.

- -Desarrollo de habilidades de reconocimiento e identificación de las conductas que constituyen los estilos de vida adecuados, así como también de los comportamientos que preceden a situaciones de riesgo.

- -Auto observación y análisis de sus propios estilos de vida. 
- -Realización de historias clínicas a pacientes con insuficiencia renal en fase dialítica.

- -Autocontrol en momentos de dispersión grupal.

- Manejo de problemáticas individuales.

- La tolerancia como herramienta para el manejo de grupos de adolescentes.

- -Desarrollo de la empatía como forma de potencializar el trabajo comunitario y la escucha.
Estas habilidades aprendidas por el grupo de trabajo son un valor agregado al aprendizaje del mismo, además de lo programado para la asignatura.

\section{Equipo responsable}

El programa fue ejecutado por profesionales de Psicología, quienes realizaron el sustento teórico del programa y los talleres. El número de estudiantes fue de 243 de VI, VIII y X semestre de Psicología.

Tabla 2. Instituciones gubernamentales, no gubernamentales y organizaciones comunitarias que apoyan la ejecución de los programas

\begin{tabular}{|c|c|c|c|c|c|}
\hline $\begin{array}{c}\text { INSTITUCIONES Y } \\
\text { ORGANIZACIONES } \\
\text { COMUNITARIAS }\end{array}$ & $\begin{array}{l}\text { REPRESENTANTE } \\
\text { LEGAL }\end{array}$ & NIT & POTENCIADA & CREADA & CAPACITADAS \\
\hline $\begin{array}{l}\text { Parroquia San Carlos } \\
\text { Borromeo }\end{array}$ & Juan Ávila & $802002940-7$ & & $\mathrm{X}$ & \\
\hline $\begin{array}{l}\text { CEB 190. Manuel Zapata } \\
\text { Olivilla. }\end{array}$ & Luz Mery Vega & $802007285-3$ & $\mathrm{X}$ & & $\mathrm{X}$ \\
\hline $\begin{array}{l}\text { CEB 200. Manuel Elkin } \\
\text { Patarroyo. }\end{array}$ & $\begin{array}{l}\text { Inocencia } \\
\text { Ramos }\end{array}$ & $802017881-6$ & $\mathrm{X}$ & & $\mathrm{X}$ \\
\hline Asociación Mis Años Bellos. & Elizabeth Vengoechea. & $9061524-3$ & & $\mathrm{X}$ & $\mathrm{X}$ \\
\hline Asociación Aguas Cristalinas. & María Clara Meza & 800140954-3 & & $\mathrm{X}$ & $\mathrm{X}$ \\
\hline $\begin{array}{l}\text { Asociación Esperanza Del } \\
\text { Por fin (madres Lactantes) }\end{array}$ & Fani Ortiz & $800245837-1$ & & $\mathrm{X}$ & $\mathrm{X}$ \\
\hline Salud Pública & $\begin{array}{c}\text { Celia Cruz Torres } \\
\text { Suárez }\end{array}$ & $890.102 .018-1$ & & & \\
\hline
\end{tabular}

\section{Actividades complementarias realizadas}

Se realizaron jornadas y actividades complementarias como las Brigadas de Salud. En éstas, el programa de Psicología coordinó la actividad con otras entidades como la Clínica de la Costa, Secretaría de Salud, CAPRECOM y las facultades que asisten a CAIFAM (Enfermería, Fisioterapia, Derecho, Trabajo Social)

También se realizaron encuentros con los niños en su día, orientados a la prevención del abuso sexual. Para esto se utilizaron actividades lúdicas, y en concreto un conversatorio con títeres, en el que participaron los estudiantes de $\mathrm{X}$ semestre Jeymi Triviño, Freddy Gómez, Sandra Pizarro y la coordinadora del programa de Psicología de la Salud, Carmen Sierra.

Se llevó a cabo el festejo del Día de la Mujer, con la participación de todos los programas y, en particular,
Psicología lo hizo con el tema de la autoestima y su importancia en el crecimiento personal.

Una última complementaria fue la socialización y sistematización de actividades formativas, en las cuales el programa de Psicología coordinó, aportando la metodología adecuada con precisión y puntualidad, así como exponiendo las acciones realizadas en el programa de Psicología de la Salud.

\section{Estrategia de la Consulta Externa}

Con respecto a la estrategia de Consulta Externa, se resalta que esta estrategia se implementaba por semestre, de marzo a 5 de junio y de agosto 15 a 5 de diciembre de cada año. Los informes ejecutivos indican que el total, desde el primer semestre del año 2000 hasta el segundo semestre de 2010, ha sido de 1808 casos.

La consulta externa se realiza de lunes a viernes, en el horario de 7:30 AM a 11:30 AM y se atiende en el 
consultorio de Psicología del Centro de Atención Integral a la Familia (CAIFAM).

Los motivos de consulta fueron en general los siguientes: el trastorno de comportamiento con $40 \%$; los trastornos de ánimo con un 20\%; trastornos por déficit de atención con hiperactividad (10\%); trastornos de aprendizaje (10\%); trastorno de relación, un 15\%; trastorno de ansiedad, el 5\%. También se presentaron aunque con mucha menos frecuencia problemas como: Trastorno relacionado con sustancias, trastorno del lenguaje expresivo, abuso sexual infantil, trastorno de la conducta alimentaria, parálisis cerebral, retardo mental, depresión infantil, síndrome de Down, estrés postraumático.

Los pacientes procedían de barrios como Los Olivos -Primera y Segunda Etapa-, Por Fin, La Manga, Edén, Calamary, Me Quejo, La Pradera, Villa Del Rosario, Las Estrellas, Sourdis, Campo Alegre, Bosque, Villas Colinas y Los Ángeles.

Por demás, se puede decir que la estrategia de consulta externa tiene muchas fortalezas en el Centro de Atención Integral a la Familia (CAIFAM), ya que la población con la cual se trabaja confía en el buen servicio prestado por los profesionales. Allí se cuenta también con un muy buen espacio físico para la realización de las consultas externas, y con materiales y recursos didácticos para la evaluación e intervención en las personas que solicitan el servicio psicológico.

Se sugiere, sin embargo, renovación del material didáctico y actualización de pruebas psicológicas. Otra debilidad que se observa es el corte de los pacientes que se realiza a mitad de año y al finalizar éste, lo cual no es conveniente, porque éstos se retiran y se presenta un cambio de estudiantes que no es lo más adecuado. Situación ésta que no les gusta a los pacientes, porque hay que iniciar de nuevo y con frecuencia los enfoques que trae el nuevo practicante son diferentes.

A la vez, se sugiere que las condiciones ambiéntales mejoren. Aun cuando cada cubículo cuenta con un ventilador, en épocas de verano el calor es insoportable. Se sugiere, por ello, la instalación de aires acondicionados, así como la adquisición de un computador por el tipo de información administrativa que se requiere recopilar. En su defecto, asignar en la Universidad Simón Bolívar un sitio donde se ubique la coordinadora para tal fin, ya que no se cuenta con este instrumento tecnológico y el trabajo pertinente debe realizarse por fuera de la institución.

\section{Conclusiones}

Se puede decir que, aunque se han visto cambios sutiles en la comunidad de hipertensos, jóvenes propensos a desarrollar diabetes, las madres comunitarias y las lactantes, es necesario seguir trabajando, debido a que se trata de una población vulnerable, es decir, expuesta al manejo inadecuado de comportamientos saludables. Esta situación que viene desde el hogar por los hábitos alimenticios inadecuados y la falta de educación en prevención y promoción de la salud para combatir hábitos insanos, se viene trasmitiendo de generación en generación.

Entre las principales características socioambientales, psicológicas, económicas y culturales de la comunidad se encuentran: un nivel socioeconómico bajo, convivencia de familias numerosas, en su mayoría no nucleares, que están expuestos a enfermedades infecciosas y no cuentan con un ambiente propicio para conservar una salud óptima. A pesar de esto, la comunidad cuenta con numerosas potencialidades que se deben aprovechar como son: el entusiasmo característico de los jóvenes, las mujeres y de la población hipertensa para conservar la salud; el interés que tienen en propiciar un cambio y la disposición para colaborar con los programas efectuados por Extensión.

La forma como se logró el aprovechamiento de esas potencialidades fue la creación de conciencia acerca de los aspectos negativos de los comportamientos y hábitos poco saludables en lo físico y mental.

Así, la población respondió a las actividades de forma adecuada, mostrando gran interés en los asuntos tratados por el grupo de trabajo, acción que contribuyó a los cambios positivos que se dieron en ella misma. Se empleó el aprendizaje participativo teniendo en cuenta la consulta, el debate y la reflexión, como medio para lograr los objetivos, ya que siempre se tuvo en cuenta el conocimiento previo que la comunidad tenía sobre el tema a tratar. Por eso, se afirma que los trabajos realizados tuvieron totalmente en cuenta a la comunidad, siendo sus miembros los propios generadores del conocimiento y ellos mismos fueron quienes se apropiaron de lo que lograban construir.

También fue un trabajo mancomunado entre el grupo de practicantes y la comunidad, pues se tuvieron 
en cuenta los parámetros recomendados por la actual psicología de la salud y los propuestos por la intervención de la psicología social de la salud, según los diferentes autores que asumen estos temas, como Flórez, (10), Brannon (11) y otros. Esto facilitó, sin duda, la implementación exitosa del programa en la comunidad.

En general, puede afirmarse que el mejoramiento de la calidad de vida en este sector del Suroccidente de Barranquilla, se ha convertido en la meta más importante que orienta el quehacer psicológico en el ámbito de la salud, no sólo en la promoción y prevención, sino en las acciones propias del tratamiento.

Las problemáticas mundiales y los cambios conceptuales de la salud, que han pasado de un abordaje epidemiológico centrado en la atención de lo físico a un abordaje más social y constructivista, abren el panorama de las acciones que todas las disciplinas deben ejercer en un constructo tan amplio como la salud (12). La Psicología, como disciplina, y en especial la Psicología de la Salud, cuenta con fortalezas que al ser contempladas por todos los agentes que participan y definen las propuestas del campo, aportan beneficios para consolidar las metas propuestas por la OMS (13). En este último sentido, pesar que la carta de Ottawa fue presentada hace ya 20 años, sus elementos principales contribuyen a entender la salud de manera integral y determinan los indicadores sobre la factibilidad y la calidad de los procesos de salud.

Dada la complejidad de la salud, su dinámica y las variables inmersas en ella, los mecanismos utilizados a nivel individual, social, comunitario y político, se enfrentan con limitantes que previenen sobre su pertinencia, factibilidad y viabilidad. Por eso, la cantidad de aspectos psicológicos involucrados en las áreas, estrategias y ambientes de la salud, ameritan la construcción de programas que sean liderados desde la academia y que incorporen a la comunidad y al trabajo interdisciplinario, interinstitucional e intersectorial.

Estos retos también exigen unas competencias teóricas y prácticas que cristalicen en propuestas para afianzar la disciplina, la construcción teórica, la participación de las comunidades y la sostenibilidad en los proyectos que se desarrollan.

\section{Referencias}

1. Riso W. Aprendiendo a quererse a sí mismo. Bogotá: Norma; 2003.

2. Figueroa $\mathrm{C}$, Ramos RB. Factores de riesgo de la hipertensión arterial y la salud cardiovascular en estudiantes universitarios. Anales de Psicología. 2006; 22 (2): 169-174.

3. Sierra C. Informe Ejecutivo. Programa Psicología de la Salud, Universidad Simón Bolívar. Localidad Sur- Occidente. Barranquilla. 2005 2010.

4. Jara HO. Sistematización de experiencias: un concepto enraizado en la realidad latinoamericana. Revista Internacional Magisterio, Educación y Pedagogía. 2008; (3):1419.

5. Martinic S. El objeto de la sistematización y sus relaciones con la evaluación y la investigación, ponencia presentada en el seminario Latinoamericano de sistematización de las prácticas de animación sociocultural y participación ciudadana en América Latina, Medellín (Colombia). 1998.

6. Doise W. Psychologie sociale et constructivisme cognitic. Archive de psychologie. 1988; 53: 127140.

7. Remy J. La transaction, une méthode d'analyse: contribution à l'émergence dún nouveau paradigme. Environnement \& Société. 1996; (17): 9-31.

8. Rodríguez M. Aspectos psicosociales de la salud y de la comunidad. Psicología social y sociedad del bienestar. III Congreso de Psicología Social. 1990; 13-26.

9. Oblitas L. Psicología de la salud y calidad de vida. Tercera edición. México: Thompson Editores; 2009.

10. Flórez AL. El proceso psicológico de la Promoción y de la Prevención. Avances en Psicología Clínica latinoamericana. 2000; 18: 1322.

11. Brannon L, Feist J. Psicología de la Salud. España: Thomson editores; 2001. 
12. Hernández A. El derecho a la salud en Colombia: obstáculos estructurales para su realización. Rev Salud Pública. 2002; 2 (2): 133-164.

13. Organización Mundial de la Salud (OMS). Informe sobre la salud en el mundo - Salud mental: nuevos conocimientos, nuevas esperanzas. 2001. Disponible en http://www.who.int/whr/2001/media_centre/es/in dex.html. 